\title{
Characterization of Compass M-1 signals
}

\author{
André Hauschild • Oliver Montenbruck • \\ Jean-Marie Sleewaegen · Lennard Huisman • \\ Peter J. G. Teunissen
}

Received: 29 September 2010/ Accepted: 21 January 2011

(c) Springer-Verlag 2011

\begin{abstract}
An analysis of observations from China's first medium earth orbit satellite Compass M-1 is presented, with main focus on the first orbit and clock solution for this satellite. The orbit is computed from laser ranging measurements. Based on this orbit solution, the apparent clock offset is estimated using measurements from two GNSS receivers, which allow Compass tracking. The analysis of the clock solutions reveals unexpectedly high dynamics in the pseudorange and carrier-phase observations. Furthermore, carrier-to-noise density ratio, pseudorange noise, and multipath are analyzed and compared to GPS and GIOVE. The results of the clock analysis motivate further research on the signals of the geostationary satellites of the Compass constellation.
\end{abstract}

Keywords Compass M-1 - Beidou 2A - Signal analysis · Orbit and clock determination

\section{Compass M-1 satellite overview}

Compass M-1, or Beidou 2A, is the first satellite of China's new global navigation satellite system (GNSS) that was

\author{
A. Hauschild $(\square) \cdot$ O. Montenbruck \\ German Space Operations Center (GSOC), Deutsches Zentrum \\ für Luft-und Raumfahrt (DLR), Wessling, Germany \\ e-mail: andre.hauschild@dlr.de \\ J.-M. Sleewaegen \\ Septentrio Satellite Navigation, Leuven, Belgium \\ L. Huisman \\ GNSS Research Centre, Curtin University of Technology, \\ Perth, Australia \\ P. J. G. Teunissen \\ DEOS, Delft University of Technology, Delft, The Netherlands
}

launched into a medium earth orbit (MEO). The complete Compass constellation will most likely consist of 30 satellites in six orbital planes in MEO and five additional satellites on geostationary or inclined geosynchronous orbits over China. Soon after the launch on April 13, 2007, researchers started to decode the satellite's PRN code sequence with directive antennas (Greilier et al. 2007; Gao et al. 2009) and to analyze the signals with software and hardware receivers (Gao et al. 2009; De Wilde et al. 2007).

The Compass M-1 satellite transmits signals on three frequencies: E2 at 1,561.098 MHz, E6 at 1,268.52 MHz, and E5b at 1,207.14 MHz. Contrary to the filings at the International Telecommunication Union (ITU), the fourth frequency $\mathrm{E} 1$ at $1,589.74 \mathrm{MHz}$ is apparently not utilized (Gao et al. 2009). Over the previous years of operation, the modulations of the satellite signals on the different frequencies have apparently been changed (Perelló Gisbert et al. 2009). Furthermore, signal anomalies have been discovered recently during the implementation of Compass tracking in a hardware receiver (Sleewaegen 2010).

Even though China has purchased a number of Rubidium clocks from the same manufacturer that also supplies the atomic clock for GIOVE and the later Galileo system, Compass M-1 has been launched before any of the units have been delivered. Instead, the four Rubidium atomic frequency standards onboard this satellite have been developed and produced in China (Mallette et al. 2010).

The orbit of Compass M-1 has an inclination of 56.3 degrees and a semimajor axis of $27,910 \mathrm{~km}$. It is approximately $1,350 \mathrm{~km}$ above the GPS constellation and about $1,700 \mathrm{~km}$ below the future Galileo satellites. The revolution period is $773 \mathrm{~min}$, leading to 1.86 revolutions per day. The ground track of the satellite repeats every 7 days. Compass M-1 is equipped with a satellite laser ranging (SLR) retroreflector and is frequently tracked by 
SLR stations around the world, which makes orbit determination possible even without global tracking of the radio navigation signals. Fig. 1 shows a drawing of the satellite (Cao et al. 2008).

\section{Reference stations and tracking equipment}

The locations of GPS/Compass capable dual-frequency receivers used in this study are shown in Fig. 2. Currently, only two stations deployed in Europe and Australia are available, which offer single-station coverage approximately $50 \%$ of the time with a $10^{\circ}$ elevation mask. A small area with dual-station coverage is available over the Arabian Sea and India.

Stations are set up at the German Space Operations Center (GSOC) of DLR in Oberpfaffenhofen, Germany, and at Curtin University of Technology (CUT), Perth, Australia. The station IDs are GSOC for the DLR station and PERX for the station at CUT. At GSOC, a Septentrio AsteRx3 receiver is connected to a Leica AR25.R3 antenna. A Septentrio PolaRx4 receiver and a Trimble TRM59800.0 choke ring antenna are used at PERX. Both receivers are equipped with a special firmware that enables Compass tracking. The signals supported by the PolaRx4 and the AsteRx 3 are identical in principle. GLONASS tracking is disabled for the AsteRx 3 receiver at GSOC, but enabled for the PolaRx4 at PERX. Furthermore, tracking of E5b signals is supported by the receiver's hardware but not enabled by the software at GSOC. The Compass M-1 satellite is tracked on the Galileo channels together with GIOVE-A and GIOVE-B with the experimental firmware. The equipment information is summarized in Table 1.

\section{Carrier-to-noise density ratio}

The carrier-to-noise density ratio over elevation as reported by the AsteRx 3 and PolarRx4 receivers for GPS, Galileo, and Compass signals is depicted in Fig. 3. A test period of 14 days from July 23 to August 7, 2010, is used to cover the ground track repetition period of both Compass (7 days) and Galileo (10 days). The left plot shows the results obtained for the GSOC station. The $\mathrm{C} / \mathrm{N}_{0}$ for GPS (plotted in black) is restricted to Block IIR-M satellites and the newest Block IIF satellite to gain more consistent results. It becomes obvious that the $\mathrm{C} / \mathrm{N}_{0}$ for $\mathrm{C} / \mathrm{A}$ code signals starts at approximately $41 \mathrm{~dB}-\mathrm{Hz}$ at an elevation angle of $5^{\circ}$ and reaches $50 \mathrm{~dB}-\mathrm{Hz}$ at an elevation angle of $30^{\circ}$. It stays close to this level also for higher elevation
Fig. 1 Drawing of the MEO satellite Compass M-1 (Cao et al. 2008)

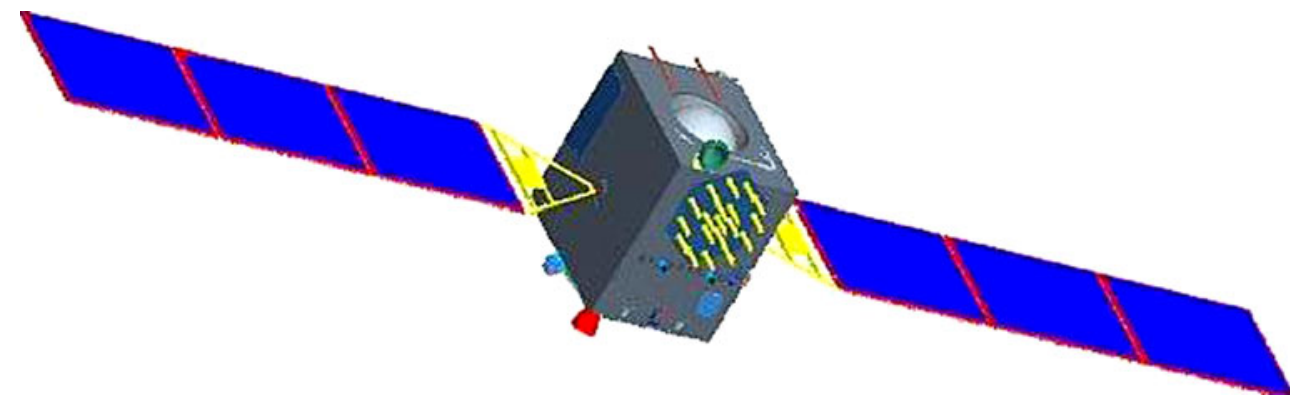

Fig. 2 Tracking network for Compass M-1 satellite. The color code indicates the number of stations that are able to track the satellite simultaneously at a given sub-satellite point

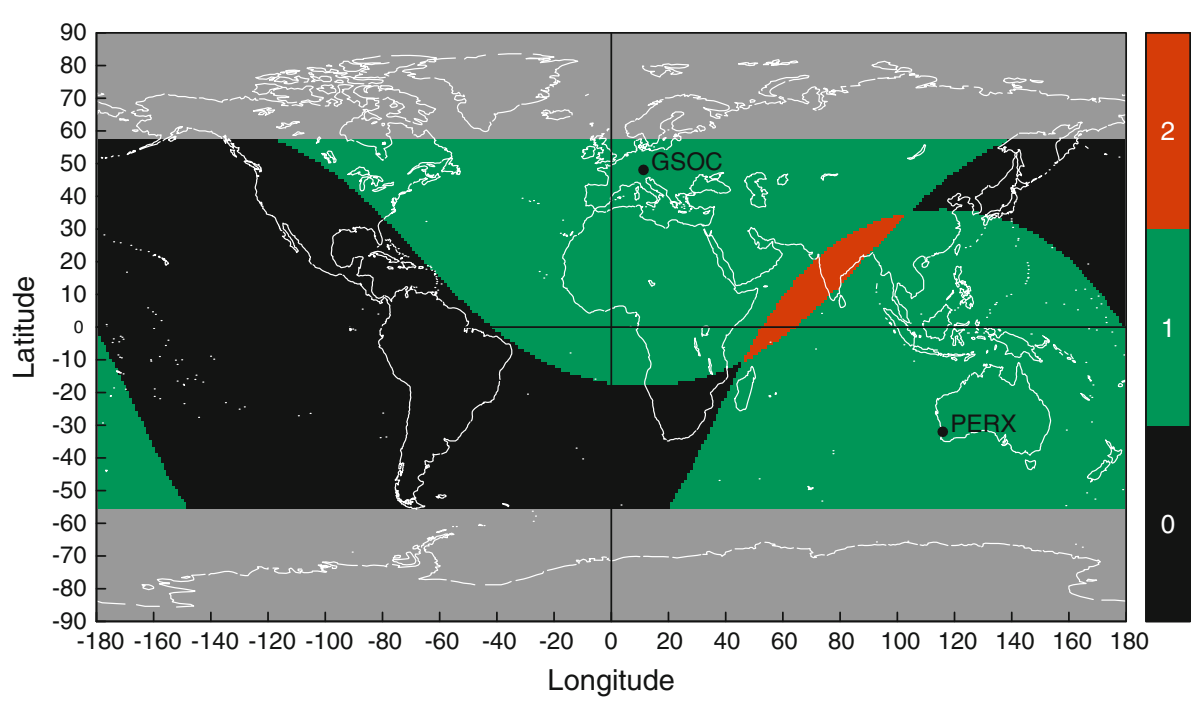


Table 1 Overview of equipment for the COMPASS tracking stations

\begin{tabular}{llll}
\hline Station & Receiver/firmware & Antenna/radome & Enabled signals \\
\hline GSOC & AsteRx3 & Leica AR25.R3 & GPS: C/A, P(Y)-L1, L2C, P(Y)-L2, L5 \\
& 2.1-tst100521r27972 & LEIT & GLO: - \\
& & & GAL: E1 Data, E5a Pilot, (E5b Pilot) \\
& & & COM: E2 Data, E5b Data \\
PERX & PolaRx4 & Trimble TRM59800.0 & GPS: C/A, P(Y)-L1, L2C, P(Y)-L2, L5 \\
& $2.1-$ tst100722r27936 & SCIS & GLO: L1, L2 \\
& & & GAL: E1 Data, E5a Pilot, E5b Pilot \\
& & & COM: E2 Data, E5b Data \\
\hline
\end{tabular}
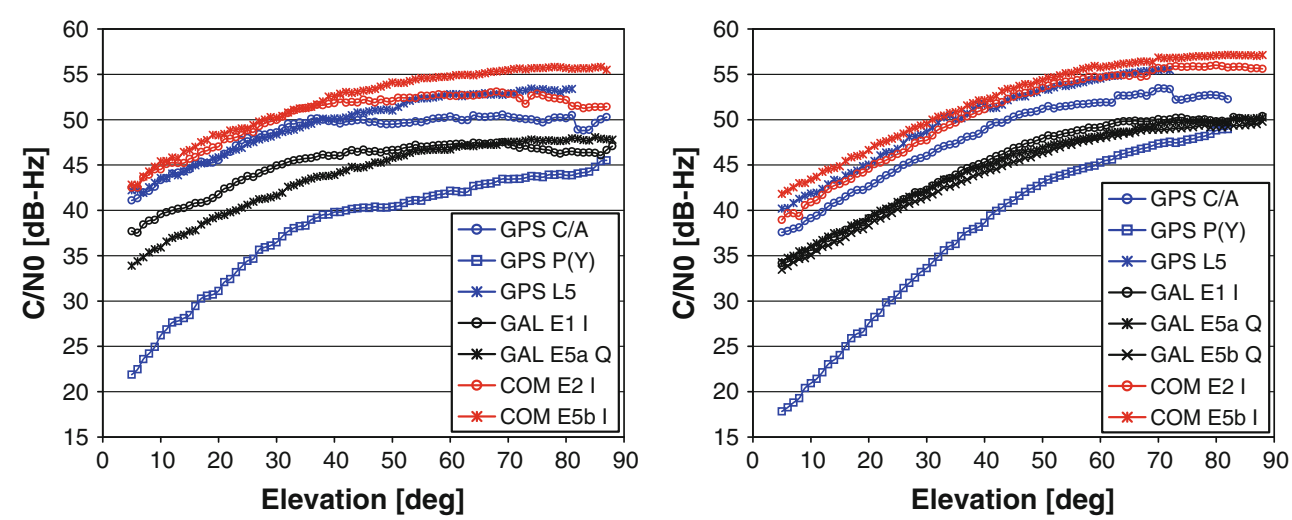

Fig. 3 Carrier-to-noise density ratios for the AsteRx3 receiver and the Leica antenna (left plot) and the PolaRx4 receiver with the Trimble antenna (right plot) for GPS (blue), GIOVE (black) and Compass M-1 (red) signals. For GPS, only Block IIRM and IIF satellites are regarded and GIOVE-B has been used exclusively. A 14 day data set has been used to cover the full ground track repetition cycle of both Compass and Galileo angles up to the zenith. Naturally, the P(Y) signal on L1 has a much lower carrier-to-noise density ratio due to the semicodeless tracking losses. The $\mathrm{C} / \mathrm{N}_{0}$ for $\mathrm{P}(\mathrm{Y})$ signals starts at about $22 \mathrm{~dB}-\mathrm{Hz}$ at low elevation angles and reaches approximately $45 \mathrm{~dB}-\mathrm{Hz}$ at zenith. The carrier-tonoise density ratio for the GPS L5 signal starts at $41 \mathrm{~dB}-\mathrm{Hz}$ at low elevation angles and rises to $53 \mathrm{~dB}-\mathrm{Hz}$ close to zenith, exceeding the $\mathrm{C} / \mathrm{N}_{0}$ for the $\mathrm{C} / \mathrm{A}$ code for an elevation angle higher than $40^{\circ}$. It should be noted that the $\mathrm{C} / \mathrm{N}_{0}$ for L5 stems from SVN 62 exclusively, which is the first Block IIF satellite transmitting a regular L5 signal.

Regarding Galileo signals, measurements from GIOVE$B$ have been used exclusively, since GIOVE-A and GIOVE-B exhibit significant differences in the transmit power levels, especially for the E5 signals. It becomes obvious that both the E1B and the E5a data signals have lower carrier-to-noise density ratios than their corresponding GPS signals. The $\mathrm{C} / \mathrm{N}_{0}$ for $\mathrm{E} 1 \mathrm{~B}$ starts at $37 \mathrm{~dB}-\mathrm{Hz}$ at low elevation, reaches a maximum of $47 \mathrm{~dB}-\mathrm{Hz}$ at $50-60^{\circ}$, and falls off by approximately $1 \mathrm{~dB}$ until zenith. The data signal on E5a, on the other hand, has a very similar variation over elevation as the GPS L5 signal, but is 6-7 dB less powerful. It should be kept in mind, however, that the signal power levels of the GIOVE satellites are most likely not representative for the future Galileo system.

Finally, the signals of the Compass M-1 satellite exhibit the highest signal-to-noise density ratio compared to all other signals. The signals on the E2 and E5b frequencies exhibit $\mathrm{C} / \mathrm{N}_{0}$ values of approximately $43 \mathrm{~dB}-\mathrm{Hz}$ at elevation $5^{\circ}$. The data signal on $\mathrm{E} 2$ reaches a maximum $\mathrm{C} / \mathrm{N}_{0}$ of $53 \mathrm{~dB}-\mathrm{Hz}$ at $50-60^{\circ}$, which reduces again to $51 \mathrm{~dB}-\mathrm{Hz}$ at zenith. The data signal on E5b rises even higher reaching a maximum carrier-to-noise density ratio of more than $55 \mathrm{~dB}-\mathrm{Hz}$.

It must be kept in mind, however, that the carrier-to-noise density ratios reported by the receiver are a result of the different gains and losses along the complete transmitting chain, including the satellite payload and antenna, the signal path, and finally the receiving antenna and receiver. Therefore, a comparison with the $\mathrm{C} / \mathrm{N}_{0}$ measurements from the station PERX reveals the effects caused by the different user equipment on the ground. Due to the communalities between the AsteRx3 and the PolaRx4, receiver-dependent differences in the reported $\mathrm{C} / \mathrm{N}_{0}$ measurements are not expected. Therefore, the differences in the carrier-to-noise density ratios are mainly caused by differences in the antenna gain. The right plot in Fig. 3 shows the reported $\mathrm{C} / \mathrm{N}_{0}$ for PERX. For the GPS C/A signal, the $\mathrm{C} / \mathrm{N}_{0}$ starts at $37 \mathrm{~dB}-\mathrm{Hz}$ at $5^{\circ}$ and reaches $53 \mathrm{~dB}-\mathrm{Hz}$ close to zenith. Compared to the Leica antenna, the $\mathrm{C} / \mathrm{N}_{0}$ of the Trimble antenna is higher close to zenith and falls off more quickly for medium and low elevation angles, especially in the E1-L1-E2 frequency band. The PolaRx4 receiver additionally provides GIOVE measurements on the E5b frequency. As expected, the $\mathrm{C} / \mathrm{N}_{0}$ for 
the pilot signals on E5a and E5b are almost identical and start at about $34 \mathrm{~dB}-\mathrm{Hz}$ at low elevation and reach $50 \mathrm{~dB}-\mathrm{Hz}$ at zenith. The carrier-to-noise density ratio of the data signal on $\mathrm{E} 1$ does not differ significantly from the results for $\mathrm{E} 5 \mathrm{a} / \mathrm{b}$, except for elevation angles between 40 and $65^{\circ}$ where it is approximately $1 \mathrm{~dB}$ higher. The PolaRx 4 receiver also reports the highest carrier-to-noise density ratio for the Compass M-1 data signal on the E5b frequency that starts at about $42 \mathrm{~dB}-\mathrm{Hz}$ and reaches up to $57 \mathrm{~dB}-\mathrm{Hz}$. The $\mathrm{C} / \mathrm{N}_{0}$ for the data signal on E2 has a similar dependency on elevation, but is about $2 \mathrm{~dB}$ lower at low elevation angles and about $1 \mathrm{~dB}$ lower at zenith.

In summary, the Leica AR25.R3 and the TRM58900.0 antenna exhibit different elevation dependencies of the antenna gains, especially for the signals in the L1 band. For both sites, the Compass M-1 signals have high carrier-tonoise density ratios, which exceed the values for the signal of GPS and GIOVE-B on the corresponding frequency bands.

\section{Noise and multipath analysis}

Figure 4 shows the results for the multipath (MP) combination of the E2 and E5b signals of Compass M-1 during a pass with elevation over the station at GSOC. The MP combination is a measure of the combined results of multipath, receiver noise, and bias variations between pseudorange and carrier-phase measurements. It has been computed according to

$M P_{\mathrm{A}}=\boldsymbol{\rho}_{\mathrm{A}}-(a+1) \cdot \Phi_{\mathrm{A}}+a \cdot \Phi_{\mathrm{B}}+b$

with

$a=2 \cdot f_{\mathrm{B}}^{2} /\left(f_{\mathrm{A}}^{2}-f_{\mathrm{B}}^{2}\right)$

where $M P_{\mathrm{A}}$ is the multipath of the signal $\mathrm{A}, \rho_{\mathrm{A}}$ and $\Phi_{\mathrm{A}}$ are pseudorange and carrier phase on signal $\mathrm{A}, \Phi_{\mathrm{B}}$ is a carrier phase on signal $\mathrm{B}$, and $\mathrm{b}$ is an arbitrary bias. The corresponding frequencies are $f_{\mathrm{A}}$ and $f_{\mathrm{B}}$. Both plots in Fig. 4 show a systematic long-term variation with superimposed stochastic errors. Whereas the latter is caused by the receiver noise and local multipath, the origin of the systematic component cannot be identified conclusively. It becomes obvious that the systematic variation is similar for both signals, but more noise is present on E2. A comparable systematic variation in the multipath combination does not exist for GPS or GIOVE.

The plot in Fig. 5 shows results of the multipath combinations for the pseudorange measurements plotted over satellite elevation. The standard deviation of the MP combination is plotted, since it does not include the systematic error component. The results for the AsteRx3 receiver at Oberpfaffenhofen are depicted in the left plot, and the PolaRx4 receiver at Perth is shown in the right plot. Again, the same test period from July 23 to August 7, 2010, is used. All observations are processed as reported by the receiver; i.e., no smoothing is applied in post-processing.
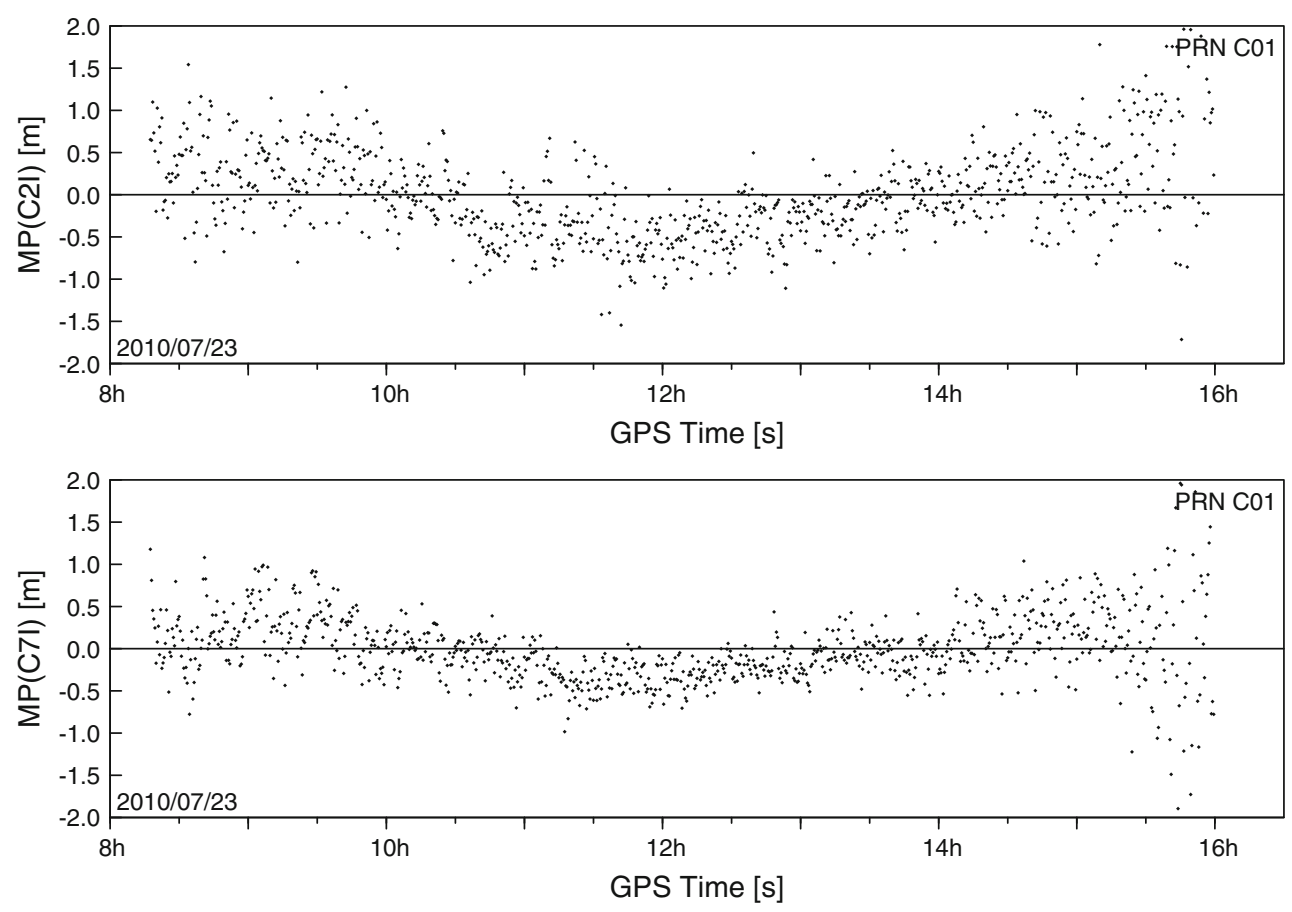

Fig. 4 Multipath combination for pseudorange on E2 (top) and E5b (bottom) of Compass M-1. The peak elevation at 11:45 UTC is approximately $80^{\circ}$ 
Fig. 5 Standard deviation of the noise and multipath error of the multipath combination for GPS, Galileo, and Compass M-1 signals for station GSOC (left plot) and PERX (right plot). For GPS, all satellites except for SVN49 (PRN01) are used. Note that only SVN62 (PRN25) contributes to the GPS L5 signal. Measurements from GIOVE-B are used for the Galileo signals. Multipath mitigation is only enabled for GPS signals
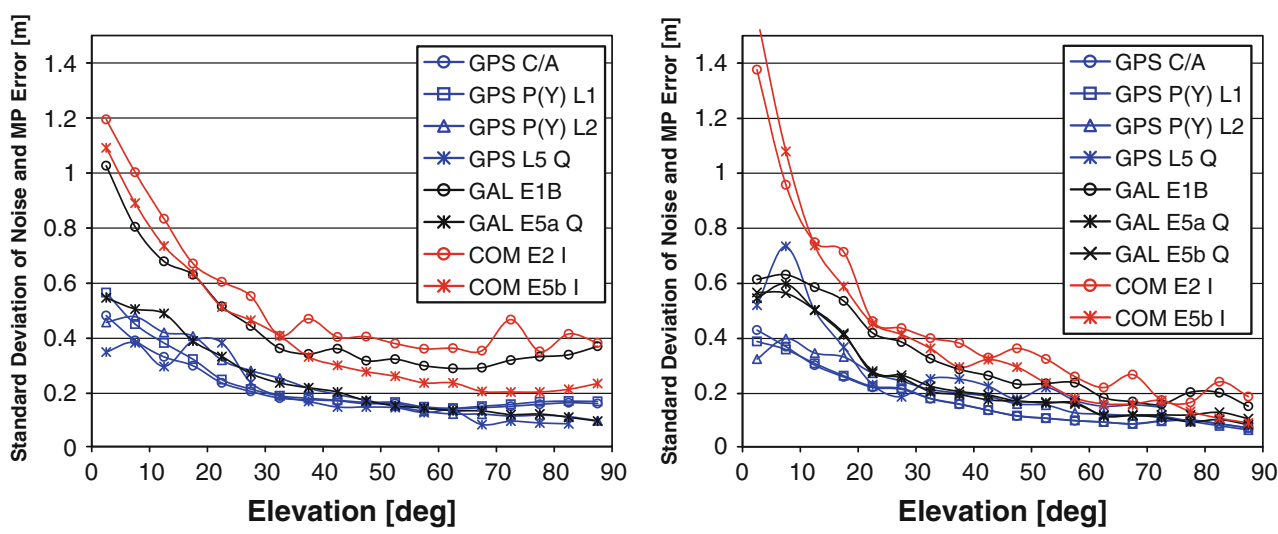

Septentrio's A-Posteriori Multipath Estimator (APME) (Sleewaegen and Boon 2001) has been activated in both receivers, but it affects only GPS C/A code in the experimental firmware. For GPS, all available satellites except for PRN01 have been processed. The GPS C/A code exhibits a standard deviation of $0.5 \mathrm{~m}$ at low elevations angles and less than $0.2 \mathrm{~m}$ at zenith. A very similar dependency can also be found for the $\mathrm{P}(\mathrm{Y})$ code on $\mathrm{L} 1$, with the exception of higher errors of about $0.55 \mathrm{~cm}$ at low elevation. The standard deviation for the $\mathrm{P}(\mathrm{Y})$ code on L2 is slightly higher for low elevation angles, but reaches a level of $0.1 \mathrm{~m}$ close to zenith. The errors for the GPS L5 signal are of the same order of magnitude as the other GPS signals. However, it should be noted that the analysis is based on the measurements of only a single satellite (SVN62/PRN25), which has almost identical observation geometry over the complete test period. For the analysis of the Galileo signals, the measurements for GIOVE-B are used. It becomes obvious that the multipath and receiver noise for the E1B signal are significantly higher compared to the pilot signal on E5a. Whereas the latter exhibits similar errors as the GPS signals, the data signal on E1 has an error of about $1 \mathrm{~m}$ at low elevation angles and about $0.4 \mathrm{~m}$ at high elevation angles. Finally, the Compass M-1 signals exhibit the largest errors for the multipath combination. Both signals show errors of more than $1 \mathrm{~m}$ at low elevation angles. The data signal on E2 and E5b still exhibit errors of about $0.2 \mathrm{~m}$ and about $0.4 \mathrm{~m}$, respectively, for high elevation angles.

The plot confirms that signals with a high chipping rate of 10.23 Mcps, which are GPS P(Y) and L5 as well as GIOVE E5a (and E5b), exhibit the smallest errors due to multipath and receiver noise. The GPS C/A code signal reaches the same performance due to multipath mitigation albeit its lower chipping rate of $1.023 \mathrm{Mcps}$. The GIOVE E1B signal with 1.023 Mcps and the two Compass signals on E2 and E5b, both with 2.046 Mcps, do not have multipath mitigation enabled. As a result, they are affected by larger multipath errors. In other analyses, the GIOVE
CBOC modulation on E1B has shown to yield comparable results to the separate modulations on E5a or E5b (Simsky et al. 2008; Montenbruck et al. 2010).

The direct comparison with the results for the PolaRx4 receiver in the right plot reveals differences caused by the different antenna environment and antenna model. The GPS signals exhibit the smallest errors again. The $\mathrm{C} / \mathrm{A}$ code and the $\mathrm{P}(\mathrm{Y})$ code on $\mathrm{L} 1$ and $\mathrm{L} 2$ reach error of less than $0.1 \mathrm{~m}$ close to zenith. The signals on E5a and E5b exhibit a comparable performance for elevation angles larger than $20^{\circ}$. For lower elevations, both signals and the GPS L5 signal are affected by larger errors, which could be related to a deficiency of the choke ring in this frequency band. The GIOVE E1B signal has a significantly better performance compared to the previous plot. However, with errors between $0.6 \mathrm{~m}$ at low elevations and $0.18 \mathrm{~cm}$ at high elevations, the multipath and receiver noise are still higher compared to the GPS signals. Like for the receiver at GSOC, the multipath mitigation is also only enabled for GPS signals. The errors of the Compass M-1 pseudoranges are highest again. However, for high elevation angles, the standard deviation of the multipath combination for the E2 and E5b signals reaches a similar level as GIOVE's E1 and E5b signals. The comparison of both tracking stations reveals that GSOC is obviously more affected by multipath reflections than PERX, which is an expected result since the antenna at GSOC is set up near several reflective surfaces.

\section{Orbit and clock determination}

Results of COMPASS M-1 orbit and clock offset estimation are presented in the following sections. In lack of a global tracking network or broadcast ephemerides for the satellite, the orbit is determined from laser ranging measurements. Based on these orbits, the computation of the clock solution is possible with the measurements from the two COMPASS tracking stations. 


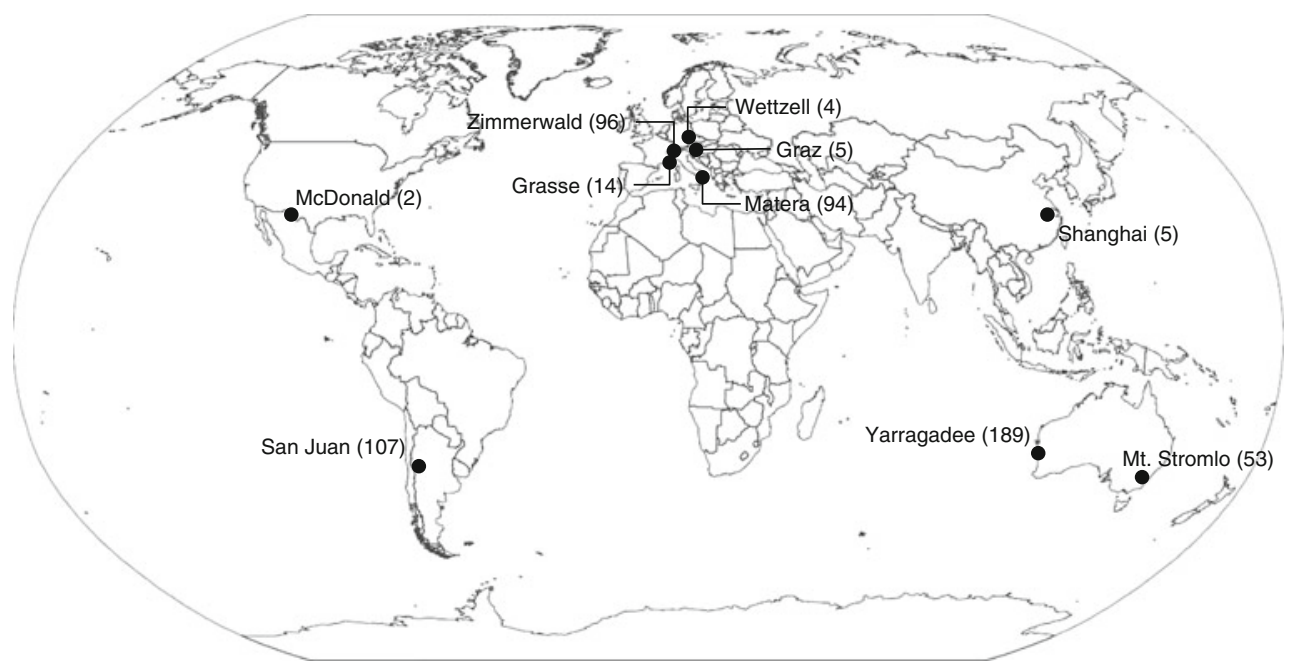

Fig. 6 Overview of satellite laser ranging stations used for Compass M-1 satellite orbit determination. Numbers in parentheses indicate the total number of normal points available during the test period from the corresponding stations

\section{SLR orbit determination}

The satellite laser ranging (SLR) measurements for Compass M-1 are obtained from ILRS (Pearlman et al. 2002). Fig. 6 depicts a map with SLR stations that have contributed measurements. The number of SLR observations, i.e., normal points, from each station during the complete test period is indicated in parenthesis. About 570 observations are available in total over the two-week period. The daily number of normal points varies between 13 and 72 .

The orbit determination is based on an iterative leastsquares filter that fits the observations to a dynamical orbit model. The state vector comprises the satellite position and velocity as well as nine radiation pressure parameters (Springer et al. 1999). A fit interval of 7 days is selected, and the orbit determination runs are performed once per day. The a-priori orbit is obtained from the orbit solution of the previous day. Earth tides, polar tides, and ocean loading are included in the modeling of the SLR station position. The satellite orbit is propagated using a simplified gravity model with 20 by 20 terms. In lack of a dedicated attitude model, Compass M-1 is assumed to follow the same attitude law as the GPS satellites.

The accuracy of the orbit determination is assessed by a comparison of trajectory overlaps. For the results in Table 2, the central day of the 7-day arc has been compared to the first day of the arc from a subsequent orbit determination run. It can be seen that the RMS of the 3-D overlap error typically varies between $11 \mathrm{~cm}$ and $50 \mathrm{~cm}$, except for the last 4 days of the test period, where the errors rise to more than $200 \mathrm{~cm}$. This increase in the overlap errors is an indication for problems in the orbit determination, which might be caused by a maneuver on one of the days in the data arc. Since no other source for
Table 2 Overlap errors of SLR orbits for Compass M-1

\begin{tabular}{llllll}
\hline Day & \# of & \multicolumn{3}{l}{ RMS overlap errors } & \\
\cline { 3 - 6 } 2010 & NP & $\begin{array}{l}\text { Radial } \\
{[\mathrm{m}]}\end{array}$ & $\begin{array}{l}\text { Along-track } \\
{[\mathrm{m}]}\end{array}$ & $\begin{array}{l}\text { Cross-track } \\
{[\mathrm{m}]}\end{array}$ & $\begin{array}{l}3-\mathrm{D} \\
{[\mathrm{m}]}\end{array}$ \\
\hline 204 & 72 & 0.013 & 0.045 & 0.102 & 0.112 \\
205 & 38 & 0.036 & 0.229 & 0.119 & 0.261 \\
206 & 27 & 0.032 & 0.113 & 0.399 & 0.416 \\
207 & 16 & 0.042 & 0.341 & 0.341 & 0.484 \\
208 & 22 & 0.022 & 0.048 & 0.173 & 0.181 \\
209 & 39 & 0.023 & 0.062 & 0.151 & 0.164 \\
210 & 40 & 0.025 & 0.237 & 0.152 & 0.283 \\
211 & 56 & 0.014 & 0.218 & 0.331 & 0.396 \\
212 & 52 & 0.013 & 0.072 & 0.261 & 0.271 \\
213 & 25 & 0.021 & 0.091 & 0.397 & 0.407 \\
214 & 17 & 0.011 & 0.17 & 0.476 & 0.506 \\
215 & 18 & 0.037 & 0.307 & 0.302 & 0.432 \\
216 & 13 & 0.143 & 0.655 & 0.675 & 0.951 \\
217 & 40 & 0.067 & 0.234 & 0.768 & 0.805 \\
218 & 55 & 0.113 & 0.197 & 1.268 & 1.288 \\
219 & 39 & 0.149 & 1.228 & 1.848 & 2.224 \\
\hline
\end{tabular}

The second column lists the total number of SLR observations available on the corresponding days. The overlap error is computed for the central day of a 7 day arc compared to the first day of a subsequent orbit determination

Compass M-1 orbits is available, a comparison for an assessment of the absolute errors of the SLR-based orbit determination is not possible. However, experience with SLR-based precise orbit determination (POD) for other navigation satellite suggests that the orbit exhibits errors of a few meters during periods with small overlap errors (Steigenberger et al. 2010). 


\section{Clock determination}

Based on the orbits obtained with SLR observations, the satellite clock offset is computed from the measurements of the receivers at GSOC and CUT. The clock estimation software (CEST) (Hauschild and Montenbruck 2009) is an offline version of DLR's real-time clock estimation system (RETICLE) (Hauschild and Montenbruck 2008). CEST has been extended to be able to process Compass measurements along with GPS and GIOVE. The software is based on a Kalman filter that processes dual-frequency code and carrier-phase measurements to estimate the satellite clock offset and drift. The state vector additionally comprises the ground station clock offsets, the wet component of the tropospheric delay, and the carrier-phase float ambiguities for each station. CEST allows the clock estimation of one or more user-selected, individual satellites, which can be GPS, GIOVE, or Compass. The clocks for the other satellites, which are not subject to estimation, are fixed and taken from the corresponding orbit and clock product. Station-related parameters like the receiver clock offset and the tropospheric zenith path delay are determined from the measurements of the "fixed" satellites as well as the "estimation" satellites. A random walk process noise model is used to compensate deviations of the true clock from the linear model. Inter-system/inter-frequency biases (ISBs) are included to enable consistent processing of GPS together with other satellite systems. ISBs are handled as constant parameters. The filter results can be smoothed by averaging a forward and a backward filter run. Furthermore, the software allows the clock estimation based on pseudorange observations only as well as on pseudorange and carrier-phase measurements.

Figure 7 depicts a Compass M-1 clock solution for July 23, 2010. The upper plot shows the clock results computed from pseudoranges only; the clock solution in the lower plot is based on pseudorange and carrier-phase measurements. Filter updates are performed every $10 \mathrm{~s}$, and an elevation limit of $10^{\circ}$ is used. A standard deviation of $2 \mathrm{~m}$ is selected for the pseudorange measurements and $5 \mathrm{~mm}$ is used for carrier phases. The process noise for the clock model has been selected to allow for a deviation of 0.70 m over $10 \mathrm{~s}$ for the offset and $0.5 \mathrm{~cm}$ over $900 \mathrm{~s}$ for the drift. The justification for the comparably large process noise on the clock phase will be given in the discussion of the results.

The satellite is observed by GSOC from about 8:30 UTC to about 15:15 UTC. PERX starts to observe the satellite about 15:00 UTC to 22:00 UTC. The tracking overlap on this day allows continuous clock estimates for more than $14 \mathrm{~h}$. The mean clock offset and drift have been removed from both plots in Fig. 7, leaving only the variation of the estimated clock with respect to the first-order polynomial. The mean clock offset referred to GPS system time on July 23 is about $-100 \mathrm{~ms}$, and the estimated mean clock drift is approximately $-0.26 \mathrm{~ns} / \mathrm{s}(-7.72 \mathrm{~cm} / \mathrm{s})$. This mean drift is one order of magnitude higher compared to the GPS satellites, but similar to GIOVE-A and GIOVE-B, which currently exhibit clock drifts of about $12.4 \mathrm{~cm} / \mathrm{s}$ and about $2.3 \mathrm{~cm} / \mathrm{s}$, respectively. Relative to the linear polynomial, the clock exhibits maximum variations between -16 and $12 \mathrm{~m}$ for pseudorange-based clock in Fig. 7. The solution based on code and phase measurements exhibits a similar variation on the same order of magnitude. A larger long-term variation with a periodicity of $8 \mathrm{~h}$ is apparently superimposed with a short-term variation with $1 \mathrm{~h}$ periodicity and smaller amplitude in both solutions. A correlation between the orbit period of $12 \mathrm{~h} 53 \mathrm{~min}$ and the clock variations is not obvious for this day. However, projections of the orbit errors on the line-of-sight will affect the clock offset estimation directly, since only single-station coverage is available most of the time.

The clock solution based on code and phase measurements in the lower plot in Fig. 7 exhibits unexpected features. Intuitively, the phase-based clock would be expected to be smoother than the pseudorange-based clock due to the higher measurements precision of the carrier-phase observables. However, the noise in the phase-based solution is not significantly lower. Furthermore, the phasebased clock exhibits a pattern of lines with a positive slope. Prior to the discussion of these effects, more details on the implementation of the carrier-phase processing for Compass shall be provided.

The inclusion of the phase observables required another modification of CEST to cope with a particularity of the Compass measurements. As reported by Sleewaegen (2010), the tracking of the Compass signals poses a challenge due to high dynamics in the pseudorange and carrierphase observables. They can be observed as atypical spikes in the Doppler frequencies, which occur stochastically every few tens of seconds and can reach up to $10 \mathrm{~Hz}$.

Similar to the receiver, the clock estimation process has to cope with these atypical dynamics of the Compass observables as well. If a Doppler spike occurs between two epochs, the resulting rapid change in the carrier-phase observations is erroneously interpreted as a cycle slip. As a temporary solution, the cycle slip detection for Compass has been deactivated in the clock estimation filter. These atypical high dynamics in the carrier-phase observations are also the reason for the increase in the process noise of the linear clock model. The large process noise for the clock offset allows the estimated clock to follow the shortterm behavior of the clock epoch by epoch, whereas the comparably low process noise for the drift allows a stable estimation of the clock's frequency offset.

To gain more insight into the unexpected pattern in the phase-based clock solution, a short sample of approximately 
Fig. 7 Compass M-1 clock solution for July 23, 2010, based on pseudoranges only (top) and pseudoranges and carrier phases (bottom). A first-order polynomial has been removed from the data
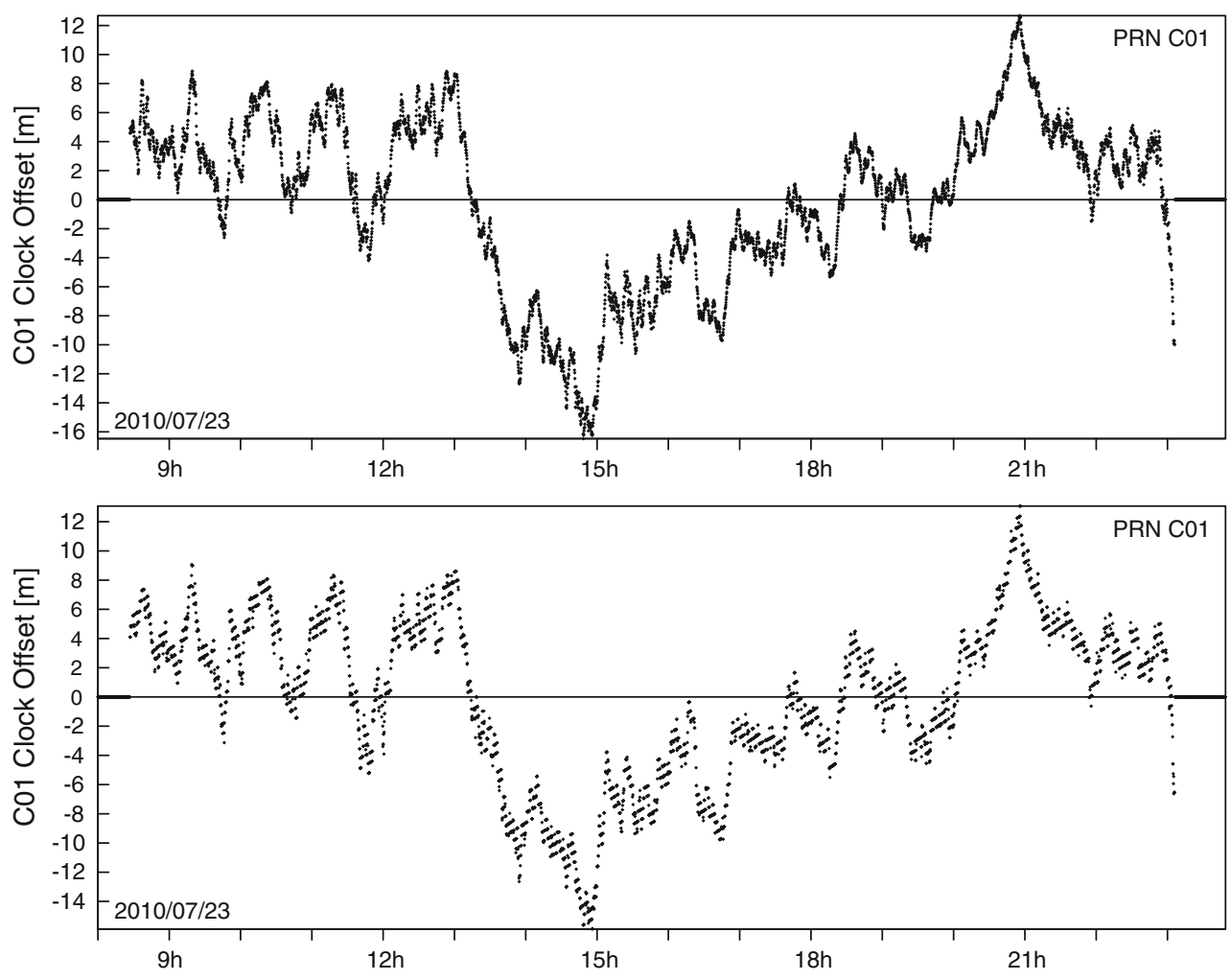

40 min is shown in Fig. 8. The clock solution in this plot is computed based on code and phase measurements with a sampling of $1 \mathrm{~Hz}$. A linear slope has again been removed. The plot reveals that the phase-based clock solution is affected by many rapid changes in the clock offset every few tens of seconds, which is consistent with the reported spikes in the Doppler frequency of the Compass satellite (Sleewaegen 2010). These changes appear as discontinuities due to the limited resolution with a sampling rate of $1 \mathrm{~Hz}$. During the short time intervals without Doppler spikes, the clock solution is comparably stable and the variation is not visible on the large scale of the plot. It is interesting to note that the mean difference of the clock offset before and after the occurrence of a Doppler spike is $67.5 \mathrm{~cm}$ with a standard deviation of $1.0 \mathrm{~cm}$. The pseudorange and carrier-phasebased clocks show very similar variations over time, which indicates that code and phase are affected in the same way. It becomes clear from Fig. 8 that the large temporal variations of the estimated clock offset reflect the accumulated changes caused by the arbitrary Doppler spikes.

It should be noted, however, that the clock estimates presented here are a measure of the "apparent" clock behavior of the satellite's atomic frequency standard. The estimated clock compensates all effects and errors, which are not included in the modeling. This includes orbit errors, possible problems in the satellite signal generation unit, unmodeled phase-center offsets and variations of the transmitting and receiving antenna, as well as unmodeled effects related to the signal path, and finally receiver tracking problems. Except for issues in the satellite clock or signal generation unit and tracking problems in the receiver, all of these effects should exhibit longer time constants and cannot be responsible for the short-term stochastic behavior of the apparent clock in Fig. 8. Under the assumption that there are no tracking problems with the receiver, a plausible explanation is instability of the satellite's onboard frequency standard, which is used for the generation of the pseudorange signal and carrier phase.

\section{Conclusions and future work}

For the analysis of Compass M-1 signals, measurements have been collected with two receivers located in Germany and Australia. China's first global navigation satellite on a MEO orbit provides a notably higher received signal power than GPS and GIOVE-B. The analysis of the multipath combination for a high-elevation ground station pass has shown that a systematic error of unknown origin is present in the measurements. The orbits for Compass have been computed from satellite laser ranging measurements with a 
Fig. 8 Forty-minute sample of Compass M-1 clock solution for July 23, 2010, based on pseudoranges and carrier phases with $1 \mathrm{~Hz}$ measurements. A first-order polynomial has been removed from the data

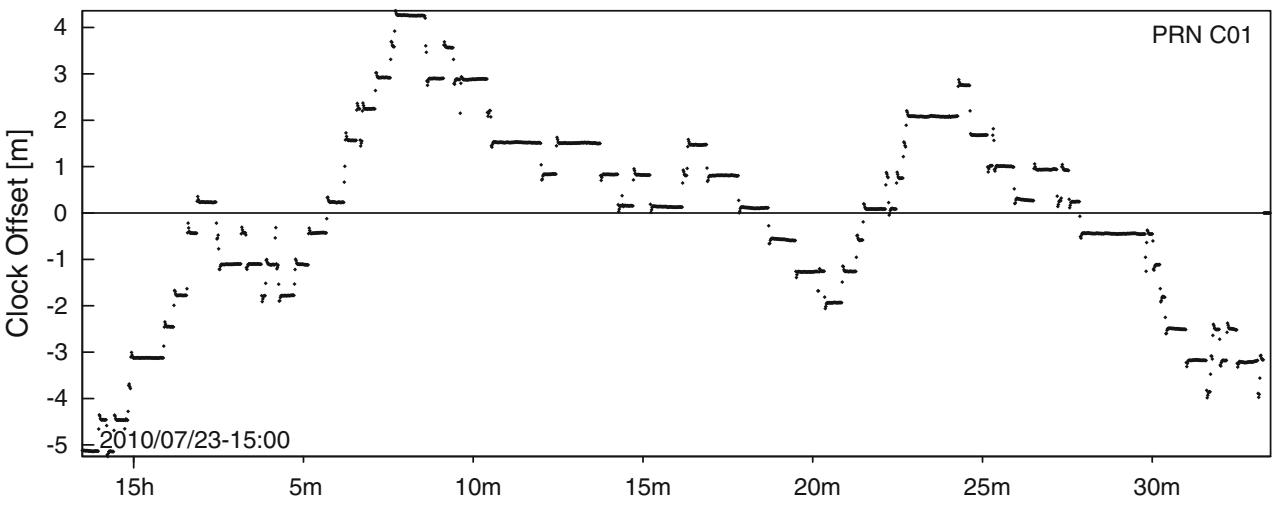

data arc of 7 days. The 3-D orbit overlap error between the first day and the middle day of two orbit solutions is typically better than $0.5 \mathrm{~m}$.

A clock solution has been computed from pseudoranges only as well as combined code measurements and phase measurements. The apparent clock solution exhibits unexpectedly high temporal variations. The results of the multipath combination and the similarity of the code and phase-based apparent clock solution show that both pseudorange and carrier-phase measurements are likewise affected by these variations. A plausible explanation for this phenomenon is that the satellite's onboard clock is subject to rapid frequency offset variations, which would affect pseudorange and carrier phase in the same way. These results are consistent with the observed Doppler spikes for the Compass M-1 satellite, which have been reported previously (Sleewaegen 2010). These random variations significantly reduce the possible accuracy of clock predictions, which would degrade the positioning accuracy of real-time users. To mitigate the effect at least partially, frequent updates of the broadcast ephemerides with low data latency would be required.

The signals of the Compass satellites on geo-stationary (GEO) or geo-synchronous (GSO) orbits are interesting subjects for further analysis. If the PRN code sequences would become available either from a release of a COMPASS interface control document or from decoding the PRN sequences, receivers could be enabled to track these satellites, allowing for the computation of orbit and clock solutions. Rubidium clocks, purchased by China from the supplier of the clocks for the future Galileo system, have been launched on two geostationary Compass satellites. According to the clock manufacturer, a comparable clock performance to the Rubidium clock of GIOVE-A can be expected for these units. An analysis of the clock solution would allow a verification of their performance. Furthermore, it would be highly interesting to check if the same phenomena, which have been observed for Compass M-1, are also present for any of the other satellites on GEO or GSO.

\section{References}

Cao C, Jing G, Luo M (2008) COMPASS satellite navigation system development. PNT challenges and opportunities symposium, Stanford, California, USA, 5-6 Nov 2008

De Wilde W, Boon F, Sleewaegen JM, Wilms F (2007) More Compass points - tracking China's MEO satellite on a hardware receiver. Inside GNSS 2(5):44-48

Gao GX, Chan A, Lo S, De Lorenzo D, Todd Walter, Enge P (2009) Compass-M1 broadcast codes in E2, E5b, and E6 frequency bands. IEEE J Sel Top Signal Process 3(4):599-612

Greilier T, Dantepal J, Delatour A, Ghion A, Enge P (2007) Initial observations and analysis of Compass MEO satellite signal. Inside GNSS 2(4):39-43

Hauschild A, Montenbruck O (2008) Real-time clock estimation for precise orbit determination of LEO-satellites. ION GNSS conference, Savannah, Georgia, 16-19 Sept 2008

Hauschild A, Montenbruck O (2009) Kalman-filter-based GPS clock estimation for near real-time positioning. GPS Solut 13(3):173182. doi:10.1007/s10291-008-0110-3

Mallette LA, White J, Rochat P (2010) Space qualified frequency sources (clocks) for current and future GNSS applications. IEEE/ ION PLANS conference 2010, Indian Wells/Palm Springs, California, USA, 4-6 May 2010

Montenbruck O, Hauschild A, Hessels U (2010) Characterization of GPS/GIOVE sensor stations in the CONGO network. GPS Solut 1-13. doi: 10.1007/s10291-010-0182-8

Pearlman MR, Degnan JJ, Bosworth JM (2002) The international laser ranging service. Adv Space Res 30(2):135-143. doi: 10.1016/S0273-1177(02)00277-6

Perelló Gisbert JV, Malik M, Rooney E, Eastment J, Davies O (2009) Selected Beidou-2A measurements and analysis. Fourth European workshop on GNSS signals and signal processing, Oberpfaffenhofen, Germany, 10-11 Dec 2009

Simsky A, Mertens D, Sleewaegen JM, De Wilde W, Hollreiser M, Crisci M (2008) Multipath and tracking performance of galileo ranging signals transmitted by GIOVE-B. ION GNSS conference, Savannah, Georgia, USA, 16-19 Sept 2008 
Sleewaegen JM (2010) 360 degrees-Compass signal quirks, another NaviForum. Inside GNSS 5(4):14-15

Sleewaegen JM, Boon F (2001) Mitigating Short-delay multipath: a promising new technique. In: Proceedings of ION GPS 2001, Salt Lake City, Utah, USA, 11-14 Sept 2001

Springer TA, Beutler G, Rothacher M (1999) A new solar radiation pressure model for GPS satellites. GPS Solut 2(3):50-62. doi: 10.1007/PL00012757

Steigenberger P, Hugentobler U, Montenbruck O, Hauschild A (2010) Precise orbit determination of GIOVE-B based on the CONGO network. J Geodesy. doi:10.1007/s00190-011-0443-5

\section{Author Biographies}
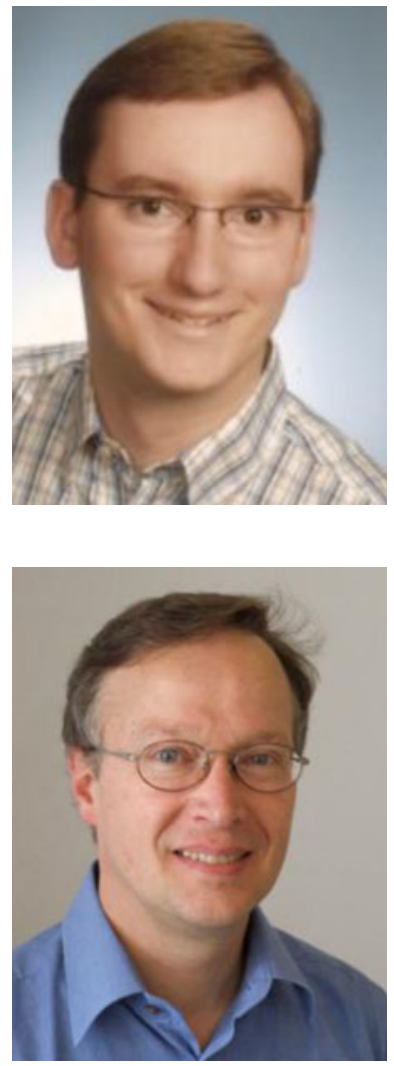

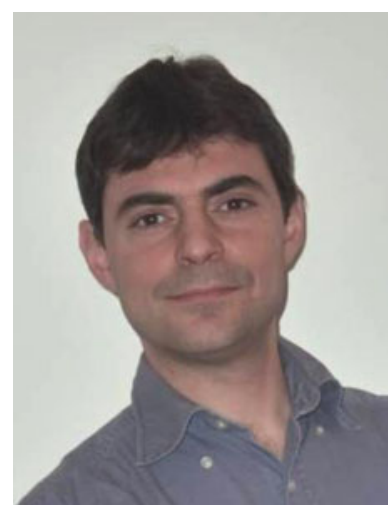

Dr. Jean-Marie Sleewaegen is currently responsible for the GNSS signal processing, system architecture and technology development at Septentrio Satellite Navigation. $\mathrm{He}$ received his $\mathrm{MSc}$ and $\mathrm{PhD}$ in electrical engineering from the University of Brussels. $\mathrm{He}$ received the Institute of Navigation (ION) Burka award in 1999.

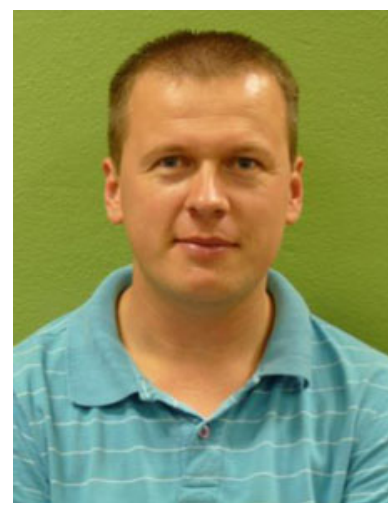

Lennard Huisman MSc is working as GNSS Engineer at the GNSS Research Centre at Curtin University, Perth, Australia. His research focuses on next-generation GNSS systems and (real-time) CORS networks. Lennard received his MSc in geodetic engineering from Delft University of Technology in 2004.

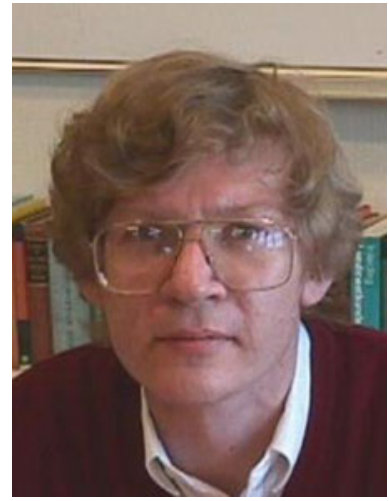

Peter J. G. Teunissen is a Federation Fellow of the Australian Research Council (ARC), Professor of Geodesy and Navigation and Head of CUT's Global Navigation Satellite System (GNSS) Research Lab. His current research focus is on modeling next-generation GNSS for relative navigation and attitude determination in space and air. $\mathrm{He}$ is the inventor of the LAMBDA method and has 25 years of GNSS research experience. 